\title{
Two sharp double inequalities for Seiffert mean
}

\author{
Yu-Ming Chu ${ }^{1 *}$, Miao-Kun Wang ${ }^{1}$ and Wei-Ming Gong ${ }^{2}$
}

* Correspondence:

chuyuming2005@yahoo.com.cn 'Department of Mathematics,

Huzhou Teachers College, Huzhou 313000, People's Republic of China Full list of author information is available at the end of the article

\section{Abstract}

In this paper, we establish two new inequalities between the root-square, arithmetic, and Seiffert means.

The achieved results are inspired by the paper of Seiffert (Die Wurzel, 29, 221-222, 1995), and the methods from Chu et al. (J. Math. Inequal., 4, 581-586, 2010). The inequalities we obtained improve the existing corresponding results and, in some sense, are optimal.

Mathematics Subject Classification (2010): $26 \mathrm{E} 60$.

Keywords: Root-square mean, arithmetic mean, Seiffert mean

\section{Introduction}

For $a, b>0$ with $a \neq b$, the root-square mean $S(a, b)$ and Seiffert mean $T(a, b)$ are defined by

$$
S(a, b)=\sqrt{\frac{a^{2}+b^{2}}{2}}
$$

and

$$
T(a, b)=\frac{a-b}{2 \arctan \left(\frac{a-b}{a+b}\right),}
$$

respectively. In the recent past, both mean values have been the subject of intensive research. In particular, many remarkable inequalities for $S$ and $T$ can be found in the literature [1-11].

Let $A(a, b)=(a+b) / 2, G(a, b)=\sqrt{a b}$, and $H(a, b)=2 a b /(a+b)$ be the classical arithmetic, geometric, and harmonic means of two positive numbers $a$ and $b$, respectively. In [1], Seiffert proved that

$$
A(a, b)<T(a, b)<S(a, b)
$$

for all $a, b>0$ with $a \neq b$.

Taneja [5] presented that

$$
\begin{aligned}
G(a, b) & <\frac{2}{3} H(a, b)+\frac{1}{3} S(a, b)<\frac{1}{2} A(a, b)+\frac{1}{2} H(a, b)<\frac{1}{2} S(a, b)+\frac{1}{2} G(a, b) \\
& <\frac{1}{3} H(a, b)+\frac{2}{3} S(a, b)<A(a, b)<S(a, b)-G(a, b)+H(a, b)
\end{aligned}
$$

for all $a, b>0$ with $\mathrm{a} \neq \mathrm{b}$. 
In [2], the authors find the greatest value $p$ and the least value $q$ such that the double inequality $H_{p}(a, b)<T(a, b)<H_{q}(a, b)$ for all $a, b>0$ with $a \neq b$. Here, $H_{p}(a, b)=\left[\left(a^{p}+(a b)^{p / 2}+b^{p}\right) / 3\right]^{1 / p}$ is the power-type Heron mean of $a$ and $b$.

Wang, Qiu, and Chu [3] established that

$$
T(a, b)<L_{1 / 3}(a, b)
$$

for all $a, b>0$ with $a \neq b$, where $L_{p}(a, b)=\left(a^{p+1}+b^{p+1}\right)=\left(a^{p}+b^{p}\right)$ is the Lehmer mean of $a$ and $b$.

The purpose of the paper is to find the greatest values $\alpha_{1}$ and $\alpha_{2}$, and the least values $\beta_{1}$ and $\beta_{2}$, such that the double inequalities $\alpha_{1} S(a, b)+\left(1-\alpha_{1}\right) A(a, b)<T(a, b)$ $<\beta_{1} S(a, b)+\left(1-\beta_{1}\right) A(a, b)$ and $S^{\alpha_{2}}(a, b) A^{1-\alpha_{2}}(a, b)<T(a, b)<S^{\beta_{2}}(a, b) A^{1-\beta_{2}}(a, b)$ hold for all $a, b>0$ with $a \neq b$.

\section{Main results}

Theorem 2.1. The double inequality $\alpha_{1} S(a, b)+\left(1-\alpha_{1}\right) A(a, b)<T(a, b)<\beta_{1} S(a, b)+$ $\left(1-\beta_{1}\right) A(a, b)$ holds for all $a, b>0$ with $a \neq b$ if and only if $\alpha_{1} \leq(4-\pi) /[(\sqrt{2}-1) \pi]=0.659 \ldots$ and $\beta_{1} \geq 2 / 3$.

Proof. Firstly, we prove that

$$
\begin{aligned}
& T(a, b)<\frac{2}{3} S(a, b)+\frac{1}{3} A(a, b) \\
& T(a, b)>\frac{4-\pi}{\pi(\sqrt{2}-1)} S(a, b)+\frac{\sqrt{2} \pi-4}{\pi(\sqrt{2}-1)} A(a, b)
\end{aligned}
$$

for all $a, b>0$ with a $\neq \mathrm{b}$.

Without loss of generality, we assume that $a>b$. Let $t=\sqrt{a / b}>1$ and $p \in\{2 / 3,(4-\pi) /[(\sqrt{2}-1) \pi]\}$, then from (1.1) and (1.2) we have

$$
\begin{aligned}
& T(a, b)-[p S(a, b)+(1-p) A(a, b)] \\
& \quad=\frac{b\left[\sqrt{2} p \sqrt{1+t^{2}}+(1-p)(1+t)\right]}{2 \arctan \left(\frac{t-1}{t+1}\right)} \\
& \quad \times\left[\frac{t-1}{\sqrt{2} p \sqrt{1+t^{2}}+(1-p)(1+t)}-\arctan \left(\frac{t-1}{t+1}\right)\right] .
\end{aligned}
$$

Let

$$
f(t)=\frac{t-1}{\sqrt{2} p \sqrt{1+t^{2}}+(1-p)(1+t)}-\arctan \left(\frac{t-1}{t+1}\right),
$$

then simple computations lead to

$$
\begin{aligned}
& f(1)=0, \\
& \lim _{t \rightarrow+\infty} f(t)=\frac{1}{(\sqrt{2}-1) p+1}-\frac{\pi}{4}, \\
& f^{\prime}(t)=\frac{f_{1}(t)}{\left(t^{2}+1\right)\left[\sqrt{2} p \sqrt{1+t^{2}}+(1-p)(1+t)\right]^{2}},
\end{aligned}
$$


where

$$
f_{1}(t)=\sqrt{2} p(2 p-1)(t+1) \sqrt{t^{2}+1}-\left[\left(3 p^{2}-1\right) t^{2}+2(p-1)^{2} t+3 p^{2}-1\right]
$$

We divide the proof into two cases.

Case 1. $p=2 / 3$. Then, we clearly see that

$$
2 p-1=3 p^{2}-1=\frac{1}{3}>0,
$$

and

$$
\begin{aligned}
& {\left[\sqrt{2} p(2 p-1)(t+1) \sqrt{1+t^{2}}\right]^{2}-\left[\left(3 p^{2}-1\right) t^{2}+2(p-1)^{2} t+3 p^{2}-1\right]^{2}} \\
& \quad=-\frac{(t-1)^{4}}{81}<0
\end{aligned}
$$

for $t>1$.

Therefore, inequality (2.1) follows from (2.3)-(2.5) and (2.7)-(2.10).

Case 2. $p=(4-\pi) /[(\sqrt{2}-1) \pi]=0.659 \ldots$. Then, simple computations yield that

$$
\begin{aligned}
& 2 p-1 i 0, \\
& 2-3 p>0, \\
& 3 p^{2}-1>0, \\
& -p^{4}-8 p^{3}+8 p^{2}-1=-0.00456 \cdots<0
\end{aligned}
$$

and

$$
\begin{aligned}
& {\left[\sqrt{2} p(2 p-1)(t+1) \sqrt{1+t^{2}}\right]^{2}-\left[\left(3 p^{2}-1\right) t^{2}+2(p-1)^{2} t+3 p^{2}-1\right]^{2}} \\
& \quad=(t-1)^{2}\left[\left(-p^{4}-8 p^{3}+8 p^{2}-1\right) t^{2}\right. \\
& \left.\quad+2\left(p^{4}-4 p^{3}+6 p^{2}-4 p+1\right) t-p^{4}-8 p^{3}+8 p^{2}-1\right] .
\end{aligned}
$$

Let

$$
\begin{aligned}
g(t)= & \left(-p^{4}-8 p^{3}+8 p^{2}-1\right) t^{2}+2\left(p^{4}-4 p^{3}+6 p^{2}-4 p+1\right) t \\
& -p^{4}-8 p^{3}+8 p^{2}-1,
\end{aligned}
$$

then from (2.11) and (2.12) together with (2.14), we get

$$
\begin{aligned}
& g(1)=4 p(2 p-1)(2-3 p)>0, \\
& \lim _{t \rightarrow+\infty} g(t)=-\infty, \\
& g^{\prime}(t)=2\left(-p^{4}-8 p^{3}+8 p^{2}-1\right) t+2\left(p^{4}-4 p^{3}+6 p^{2}-4 p+1\right), \\
& g^{\prime}(1)=4 p(2 p-1)(2-3 p)>0, \\
& \lim _{t \rightarrow+\infty} g^{\prime}(t)=-\infty
\end{aligned}
$$

and

$$
g^{\prime \prime}(t)=2\left(-p^{4}-8 p^{3}+8 p^{2}-1\right)=-0.00912 \cdots<0 .
$$


It follows from (2.19)-(2.21) that there exists $t_{0}>1$ such that $g^{\prime}(t)>0$ for $t \in\left[1, t_{0}\right)$ and $g^{\prime}(t)<0$ for $t \in\left(t_{0}, \infty\right)$. Hence, $g(t)$ is strictly increasing in $\left[1, t_{0}\right]$ and strictly decreasing in $\left[t_{0}, \infty\right)$.

From (2.17) and (2.18) together with the piecewise monotonicity of $g(t)$, we clearly see that there exists $t_{1}>t_{0}>1$ such that $g(t)>0$ for $t \in\left[1, t_{1}\right)$ and $g(t)<0$ for $t \in$ $\left(t_{1}, \infty\right)$. Then, from (2.8), (2.11), (2.13), (2.15), and (2.16), we know that $f_{1}(t)>0$ for $t \in$ $\left[1, t_{1}\right)$ and $f_{1}(t)<0$ for $t \in\left(t_{1}, \infty\right)$. It follows from $(2.7)$ that $f(t)$ is strictly increasing in $\left[1, t_{1}\right]$ and strictly decreasing in $\left[t_{1}, \infty\right)$.

Note that (2.6) becomes

$$
\lim _{t \rightarrow+\infty} f(t)=0
$$

Therefore, inequality (2.2) follows from (2.3)-(2.5) and (2.22) together with the piecewise monotonicity of $f(t)$.

Secondly, we prove that $2 S(a, b) / 3+A(a, b) / 3$ is the best possible upper convex combination bound of root-square and arithmetic means for the Seiffert mean $T(a, b)$.

Letting $x>0(x \rightarrow 0)$ and making use of the Taylor expansion, one has

$$
\begin{aligned}
\beta_{1} S & (1,1+x)+\left(1-\beta_{1}\right) A(1,1+x)-T(1,1+x) \\
& =\beta_{1}\left[\left[1+\frac{1}{2} x+\frac{1}{8} x^{2}+o\left(x^{2}\right)\right]+\left(1-\beta_{1}\right)\left(1+\frac{x}{2}\right)\right. \\
& -\left[1+\frac{1}{2} x+\frac{1}{12} x^{2}+o\left(x^{2}\right)\right] \\
& =\frac{1}{24}\left(3 \beta_{1}-2\right) x^{2}+o\left(x^{2}\right) .
\end{aligned}
$$

Equation (2.23) implies that for any $\beta_{1}<2 / 3$, there exists $\delta_{1}=\delta_{1}\left(\beta_{1}\right)>0$, such that $\beta_{1} S(1,1+x)+\left(1-\beta_{1}\right) A(1,1+x)<T(1,1+x)$ for $x \in\left(0, \delta_{1}\right)$.

Finally, we prove that $(4-\pi) S(a, b) /[(\sqrt{2}-1) \pi]+(\sqrt{2} \pi-4) A(a, b) /[(\sqrt{2}-1) \pi]$ is the best possible lower convex combination bound of root-square and arithmetic means for the Seiffert mean $T(a, b)$.

For any $\alpha_{1}>(4-\pi) /[(\sqrt{2}-1) \pi]$, it follows from (1.1) and (1.2) that

$$
\lim _{x \rightarrow+\infty} \frac{\alpha_{1} S(1, x)+\left(1-\alpha_{1}\right) A(1, x)}{T(1, x)}=\frac{(\sqrt{2}-1) \alpha_{1}+1}{4} \pi>1 .
$$

Inequality (2.24) implies that for any $\alpha_{1}>(4-\pi) /[(\sqrt{2}-1) \pi]$, there exists $X_{1}=X_{1}$ $\left(\alpha_{1}\right)>1$ such that $\alpha_{1} S(1, x)+\left(1 \alpha_{1}\right) A(1, x)>T(1, x)$ for $x \in\left(X_{1}, \infty\right)$.

Theorem 2.2. The double inequality $S^{\alpha_{2}}(a, b) A^{1-\alpha_{2}}(a, b)<T(a, b)<S^{\beta_{2}}(a, b) A^{1-\beta_{2}}(a, b)$ holds for all $a, b>0$ with $a \neq b$ if and only if $\alpha_{2} \leq 2 / 3$ and $\beta_{2} \geq 4-2 \log =\log 2=0.697 \ldots$.

Proof. Firstly, we prove that

$$
\begin{aligned}
& T(a, b)>S^{2 / 3}(a, b) A^{1 / 3}(a, b), \\
& T(a, b)<[S(a, b)]^{4-2 \log \pi / \log 2}[A(a, b)]^{2 \log \pi / \log 2-3}
\end{aligned}
$$

for all $a, b>0$ with $a \neq b$.

Without loss of generality, we assume that $a>b$. Let $t=\sqrt{a / b}>1$ and $q \in\{2 / 3,4-2$ $\log \pi / \log 2\}$, then from (1.1) and (1.2), we have 


$$
\begin{aligned}
& \log T(a, b)-[q \log S(a, b)+(1-q) \log A(a, b)] \\
& \quad=\log \frac{t-1}{2 \arctan \left(\frac{t-1}{t+1}\right)}-\frac{q}{2} \log \left(\frac{1+t^{2}}{2}\right)-(1-q) \log \left(\frac{1+t}{2}\right)
\end{aligned}
$$

Let

$$
F(t)=\log \frac{t-1}{2 \arctan \left(\frac{t-1}{t+1}\right)}-\frac{q}{2} \log \left(\frac{1+t^{2}}{2}\right)-(1-q) \log \left(\frac{1+t}{2}\right),
$$

then simple computations lead to

$$
\begin{aligned}
& \lim _{t \rightarrow 1} F(t)=0, \\
& \lim _{t \rightarrow+\infty} F(t)=\log \frac{4}{\pi}-\frac{q}{2} \log 2, \\
& F^{\prime}(t)=\frac{(2-q) t^{2}+2 q t+2-q}{\left(t^{4}-1\right) \arctan \left(\frac{t-1}{t+1}\right)} F_{1}(t)
\end{aligned}
$$

where

$$
\begin{aligned}
& F_{1}(t)=\arctan \left(\frac{t-1}{t+1}\right)-\frac{t^{2}-1}{(2-q) t^{2}+2 q t+2-q} \\
& F_{1}(1)=0 \\
& \lim _{t \rightarrow+\infty} F_{1}(t)=\frac{\pi}{4}-\frac{1}{2-q} \\
& F_{1}^{\prime}(t)=\frac{(t-1)^{2}}{\left(1+t^{2}\right)\left[(2-q) t^{2}+2 q t+2-q\right]^{2}} F_{2}(t)
\end{aligned}
$$

where

$$
\begin{aligned}
& F_{2}(t)=\left(q^{2}-6 q+4\right) t^{2}-2 q^{2} t+q^{2}-6 q+4, \\
& F_{2}(1)=4(2-3 q), \\
& F_{2}^{\prime}(t)=2\left(q^{2}-6 q+4\right) t-2 q^{2}, \\
& F_{2}^{\prime}(1)=4(2-3 q) .
\end{aligned}
$$

We divide the proof into two cases.

Case 1. $q=2 / 3$. Then, we clearly see that

$$
\begin{aligned}
& 2-3 q=0 \\
& q^{2}-6 q+4=\frac{4}{9}>0 .
\end{aligned}
$$

From (2.38)-(2.41), we know that $F_{2}^{\prime}(t)>0$ for $\mathrm{t} \in(1, \infty)$. Hence, $F_{2}(t)$ is strictly increasing in $[1, \infty)$. It follows from $(2.35),(2.37),(2.40)$, and the monotonicity of $F_{2}(t)$ that $F_{1}(t)$ is strictly increasing in $[1, \infty)$. 
Therefore, inequality (2.25) follows from (2.27)-(2.29), (2.31), (2.33), and the monotonicity of $F_{1}(t)$.

Case 2. $q=4-2 \log \pi / \log 2=0: 697 \ldots$. Then, simple computations lead to

$$
\begin{aligned}
& \log \frac{4}{\pi}-\frac{q}{2} \log 2=0, \\
& \frac{\pi}{4}-\frac{1}{2-q}=0.0179 \cdots>0, \\
& q^{2}-6 q+4=0.303 \cdots>0, \\
& 2-3 q=-0.09102 \cdots<0 .
\end{aligned}
$$

It follows from (2.36) and (2.38) together with (2.44) that

$$
\begin{aligned}
& \lim _{t \rightarrow+\infty} F_{2}(t)=+\infty, \\
& \lim _{t \rightarrow+\infty} F_{2}^{\prime}(t)=+\infty .
\end{aligned}
$$

From (2.38) and (2.44), we clearly see that $F_{2}^{\prime}(t)$ is strictly increasing in $[1, \infty)$. Then, (2.39) and (2.45) together with (2.47) imply that there exists $\lambda_{0}>1$ such that $F_{2}^{\prime}(t)<0$ for $t \in\left[1, \lambda_{0}\right)$ and $F_{2}^{\prime}(t)>0$ for $t \in\left(\lambda_{0}, \infty\right)$. Hence, $F_{2}(t)$ is strictly decreasing in [1, $\left.\lambda_{0}\right]$ and strictly increasing in $\left[\lambda_{0}, \infty\right)$.

From (2.37), (2.45), (2.46), and the piecewise monotonicity of $F_{2}(t)$, we know that there exists $\lambda_{1}>\lambda_{0}>1$ such that $F_{2}(t)<0$ for $t \in\left[1, \lambda_{1}\right)$ and $F_{2}(t)>0$ for $t \in\left(\lambda_{1}, \infty\right)$. Then (2.35) implies that $F_{1}(t)$ is strictly decreasing in $\left[1, \lambda_{1}\right]$ and strictly increasing in $\left[\lambda_{1}, \infty\right)$.

From (2.33), (2.34), (2.43), and the piecewise monotonicity of $F_{1}(t)$, we conclude that there exists $\lambda_{2}>\lambda_{1}>1$ such that $F_{1}(t)<0$ for $t \in\left(1, \lambda_{2}\right)$ and $F_{1}(t)>0$ for $t \in\left(\lambda_{2}, \infty\right)$. Then, (2.31) implies that $F(t)$ is strictly decreasing in $\left(1, \lambda_{2}\right]$ and strictly increasing in $\left[\lambda_{2}, \infty\right)$.

Therefore, inequality (2.26) follows from (2.27)-(2.30) and (2.42) together with the piecewise monotonicity of $F(t)$.

Secondly, we prove that $S^{2 / 3}(a, b) A^{1 / 3}(a, b)$ is the best possible lower geo-metric combination bound of root-square and arithmetic means for the Seiffert mean $T(a, b)$.

Letting $x>0(x \rightarrow 0)$ and making use of the Taylor expansion, one has

$$
\begin{aligned}
S^{\alpha_{2}} & (1,1+x) A^{1-\alpha_{2}}(1,1+x)-T(1,1+x) \\
& =\left[1+\frac{\alpha_{2}}{2} x+\frac{\alpha_{2^{2}}}{8} x^{2}+o\left(x^{2}\right)\right]\left[1+\frac{1-\alpha_{2}}{2} x+\frac{\alpha_{2}\left(\alpha_{2}-1\right)}{8} x^{2}+o\left(x^{2}\right)\right] \\
& -\left[1+\frac{1}{2} x+\frac{1}{12} x^{2}+o\left(x^{2}\right)\right] \\
& =\frac{1}{24}\left(3 \alpha_{2}-2\right) x^{2}+o\left(x^{2}\right) .
\end{aligned}
$$

Equation (2.48) implies that for any $\alpha_{2}>2 / 3$, there exists $\delta_{2}=\delta_{2}\left(\alpha_{2}\right)>0$, such that $S^{\alpha_{2}}(1,1+x) A^{1-\alpha_{2}}(1,1+x)>T(1,1+x)$ for $x \in\left(0, \delta_{2}\right)$.

Finally, we prove that $[S(a, b)]^{4-2 \log \pi / \log 2}[A(a, b)]^{2 \log \pi / \log 2-3}$ is the best possible upper geometric combination bound of root-square and arithmetic means for the Seiffert mean $T(a, b)$. 
For any $\beta_{2}<4-2 \log \pi / \log 2$ and $x>0$, from (1.1) and (1.2), one has

$$
\lim _{x \rightarrow+\infty} \frac{S^{\beta_{2}}(1, x) A^{1-\beta_{2}}(1, x)}{T(1, x)}=2^{\beta_{2} / 2} \times \frac{\pi}{4}<1 .
$$

Inequality (2.49) implies that for any $\beta_{2}<4-2 \log \pi / \log 2$, there exists $X_{2}=X_{2}\left(\beta_{2}\right)$ $>1$ such that $T(1, x)>S^{\beta_{2}}(1, x) A^{\left(1-\beta_{2}\right)}(1, x)$ for $x \in\left(X_{2},+\infty\right)$.

\section{Acknowledgements}

This study is partly supported by the Natural Science Foundation of China (Grant no. 11071069), the Natural Science Foundation of Hunan Province (Grant no. 09JJ6003), and the Innovation Team Foundation of the Department of Education of Zhejiang Province(Grant no. T200924).

\section{Author details}

'Department of Mathematics, Huzhou Teachers College, Huzhou 313000, People's Republic of China ${ }^{2}$ Department of Mathematics, Hunan City University, Yiyang 413000, People's Republic of China

\section{Authors' contributions}

Y-MC carried out the proof of Theorme 2.1 in this paper. M-KW carried out the proof of Theorem 2.2 in this paper. WMG provieded the main idea of this paper. All authors read and approved the final manuscript.

\section{Competing interests}

The authors declare that they have no competing interests.

Received: 12 May 2011 Accepted: 2 September 2011 Published: 2 September 2011

\section{References}

1. Seiffert, H-J: Aufgabe $\beta$ 16. Die Wurzel. 29, 221-222 (1995)

2. Chu, Y-M, Wang, M-K, Qiu, Y-F: An optimal double inequality between power-type Heron and Seiffert mean. J Inequal Appl 11 (2010). Article ID 146945

3. Wang, M-K, Qiu, Y-F, Chu, Y-M: Sharp bounds for Seiffert means in terms of Lehmer means. J Math Inequal. 4(4), $581-586$ (2010)

4. Shi, H-N, Zhang, J, Li, D-M: Schur-geometric convexity for differences of means. Appl Math E-Notes. 10, 275-284 (2010)

5. Taneja, IJ: Refinement of inequalities among means. J Comb Inf Syst Sci. 31(1-4), 343-364 (2006)

6. Nelsen, RB: Proof without words: the harmonic mean-geometric mean-arithmetic mean-root mean square inequality. Math Mag. 60(3), 158-158 (1987)

7. Chandra, J, Ladde, GS, Lakshmikantham, V: Stochastic analysis of a compressible gas lubrication slider bearing problem. SIAM J Appl Math. 43(5), 1175-1186 (1983)

8. Hoehn, L, Niven, I: Averages on the move. Math Mag. 58(3), 151-156 (1985). doi:10.2307/2689911

9. David, HA: General bounds and inequalities in order statistics. Comm Statist Theory Methods. 17(7), 2119-2134 (1988). doi:10.1080/03610928808829737

10. Hardy, GH, Littlewood, JE, Pólya, JE: Inequalities. Cambridge University Press, Cambridge (1988)

11. Huntington, EV: Sets of independent postulates for the arithmetic mean, the geometric mean, the harmonic mean, and the root-mean-square. Trans Amer Math Soc. 29(1), 1-22 (1927). doi:10.1090/S0002-9947-1927-1501374-0

doi:10.1186/1029-242X-2011-44

Cite this article as: Chu et al: Two sharp double inequalities for Seiffert mean. Journal of Inequalities and Applications 2011 2011:44.

\section{Submit your manuscript to a SpringerOpen ${ }^{\odot}$ journal and benefit from:}

- Convenient online submission

- Rigorous peer review

- Immediate publication on acceptance

- Open access: articles freely available online

- High visibility within the field

- Retaining the copyright to your article

Submit your next manuscript at $\gg$ springeropen.com 